\title{
Nanotheranostics
}

2017; 1(4): 430-439. doi: 10.7150/ntno.19935

Research Paper

\section{Nano-sized Indocyanine Green J-aggregate as a One-component Theranostic Agent}

\author{
Renfa Liu, Jie Tang, Yunxue Xu, Yiming Zhou, Zhifei Dai ${ }^{\bowtie}$ \\ Department of Biomedical Engineering, College of Engineering, Peking University, Beijing 100871, China \\ $\triangle$ Corresponding author: E-mail: zhifei.dai@pku.edu.cn \\ (c) Ivyspring International Publisher. This is an open access article distributed under the terms of the Creative Commons Attribution (CC BY-NC) license \\ (https://creativecommons.org/licenses/by-nc/4.0/). See http://ivyspring.com/terms for full terms and conditions.
}

Received: 2017.05.19; Accepted: 2017.10.09; Published: 2017.11.01

\begin{abstract}
The development of biocompatiable efficient photothermal coupling agent (PCA) for image-guided photothermal therapy of cancer has gained increasing interests in recent years. Although various PCAs have been developed, the clinical translations of these materials have been largely hindered by the potential biosafety issues and challenges of scaling-up manufactures. In this research, we proposed nano-sized indocyanine green (ICG) J-aggregate (IJA) as a promising PCA which is fabricated by a very facile method using clinical-approved ICG as the only excipient. The as-prepared IJA remains stable in various solution and shows a $\sim 115 \mathrm{~nm}$ red-shift in absorption peak compared to free ICG. Importantly, IJA can be disassociated into free ICG again after internalized into cells and exhibits high biosafety comparable to ICG. The IJA performs well for photothermal therapy both in vitro and in vivo. In addition, the IJA can also be used as a good photoacoustic contrast agent and internalization-responsive fluorescence probe. The facile preparation, high safety and excellent theranostic performance indicated that IJA might be a promising one-component agent for cancer theranostics.
\end{abstract}

Key words: Indocyanine green, J-aggregate, photothermal therapy, theranostics, photoacoustic imaging.

\section{Introduction}

Thermal therapy has emerged as a promising minimally invasive alternative to conventional approaches, such as surgery, chemotherapy and radiotherapy, for treatments of several tumors [1, 2]. In particular, photothermal therapy (PTT) induced by near-infrared (NIR) laser featured by lower tissue absorption and deeper penetration has attracted increasing attention in the past decade [3]. One of the most important prerequisites for successful clinical translation of PTT is to develop a clinically-acceptable efficient photothermal coupling agent (PCA).

Currently, various inorganic or organic nanomaterials have been investigated as PCA, such as noble-metal-based nanostructures [3-7], carbon nanomaterials [8-11], semiconductor nanomaterials [12-14] and conjugated polymers [15, 16]. However, most of these materials are either non-degradable or degraded into potentially hazardous metal ions in vivo
[17-19]. The long-term biosafety in vivo after systematic injection is a major concern [20, 21]. In addition, the issues in scale-up of manufacturing process is another big challenge for clinical translation [22].

Fabricating efficient PCA utilizing clinicallyapproved pharmaceutical excipients may be a shortcut for the translation of PCA. Indocyanine green (ICG) is currently the only pharmaceutical excipient with NIR absorption approved by the US Food and Drug Administration (FDA). However, the limited accumulation in vivo and high photobleaching make ICG hardly directly used for PTT. Therefore, a lot of researches have been done to improve the in vivo behavior and photothermal conversion ability using several clinically-applicable drug delivery systems, such as liposomes [23], polymers [24], lipid nanoparticles [25, 26], albumin nanoparticles [27, 28], 
nanofibers [29] and so on. However, these systems still more or less encounter problems involving low drug loading content, low photothermal conversion efficacy and complex preparation method, thus hindering their clinical translation.

Many organic dyes could self-assemble in to J-type aggregates under certain circumstances featured by a red-shifted, intense and narrow absorption peak, known as a J-band. Since its discovery in 1930s [30], enormous researches have been done concerning its distinct optical properties and several applications have been proposed [31-34]. In recent years, there are also some reports utilizing nano-sized J-aggregates of organic dyes for the theranostic applications of cancer [35-38]. As a FDA-approved organic dye, ICG was found to form J aggregates in aqueous solution under heating two decades ago [39]. Despite its distinct optical properties [39-42], the potentials of ICG J-aggregate (IJA) for biophotonic imaging and therapy in vivo have been ignored.

In this research, we proposed nano-sized IJA as a promising PCA characterized with facile preparation, excellent photothermal conversion ability and high biocompatibility (Scheme 1). Nano-sized IJA can be separated from heated ICG aqueous solution by filtration. Once forming, IJA remains stable in J-aggregated state in various media. The IJA shows excellent performance for PTT and photoacoustic imaging (PAI) both in vitro and in vivo. In addition, after internalized by cells, IJA can be disassociated into ICG monomers, exhibiting high biocompatibility similar to ICG and potential for internalizationresponsive fluorescence imaging. The facile preparation, high biocompatibility and excellent theranostic performance make the IJA a highly promising PCA for future applications.

\section{Materials and Methods}

\section{Materials and equipment}

All reagents obtained commercially were used without further purification. Indocyanine Green (ICG) was obtained from Shenyang Bomei Pharmaceutical New Technology Development Co., Ltd. $0.22 \mu \mathrm{m}$ syringe filters were bought from Sartorius. Dialysis bag (8000-14000 Da) was obtained from Beijing Solarbio Science \& Technology Co., Ltd. Millipore quality deionized water (DI water) (resistivity is 18.2 $\mathrm{M} \Omega \mathrm{cm}$ ) was utilized in all experiments. Pluronic F127 (PF127) was obtained from BASF. Polyoxyethylene-40 stearate (PEG40S), Tween 80 and Triton X-100 were obtained from Sigma Aldrich. All the other solvents used in the research were purchased from Sinopharm Chemical Reagent Beijing Co., Ltd. or Beijing (China) Chemical Company. The freeze-drying process was conducted on an Advantage 2.0 EL freeze dryer(Virtis). UV-vis-NIR spectra was recorded using an Evolution 220 UV-vis-NIR spectrophotometer (Thermal Scientific). The hydrodynamic diameter was determined with a PALS/90Plus dynamic light scattering (DLS) analyzer (Brookhaven Instruments Co., USA). The morphology of the aggregates was observed by Tecnai G2 T20 transmission electron microscopy. Photoacoustic images and signal data were measured and analyzed using a multi-spectral optoacoustic tomographic (MSOT) imaging system (inVision 128, iThera Medical GmbH, Neuherberg, Germany). The fluorescence imaging in vivo was conducted on a Maestro 2 in-vivo imaging system (Cambridge Research \& Instrumentation, Inc.). The temperature changes in vivo were monitored with an IR thermal camera (Fluke Ti27).

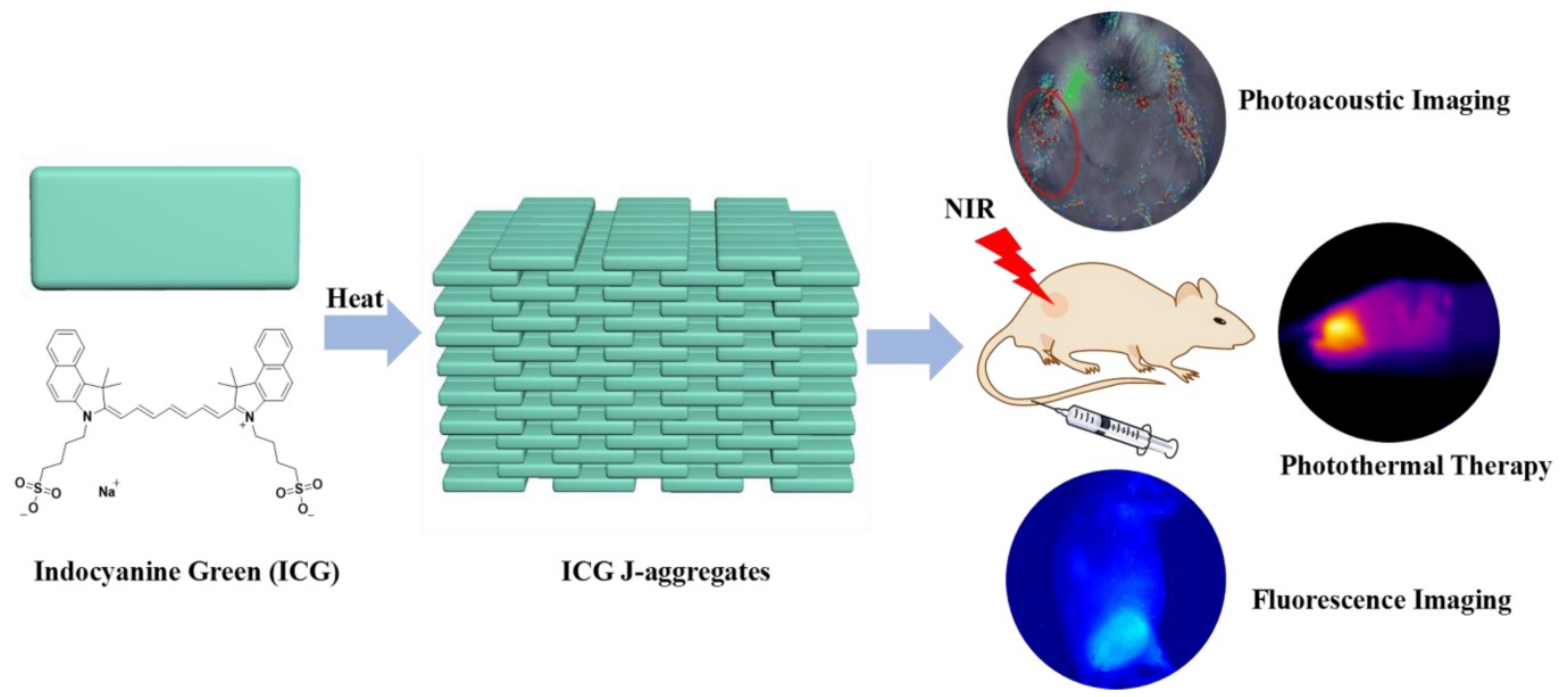

Scheme 1. Schematic illustration of the formation of IJA and its applications for photoacoustic / fluorescence imaging-guided photothermal therapy. 


\section{Preparation of ICG J-aggregates (IJA)}

$1.5 \mathrm{mM}$ ICG aqueous solution was heated in a water bath at $65^{\circ} \mathrm{C}$ for $20 \mathrm{~h}$. This process is monitored with UV-vis-NIR spectrophotometer. After the formation of J-aggregates, the solution was dialyzed against DI water for $24 \mathrm{~h}$ to remove any possible free ICG. After dialysis, the solution was filtered through $0.22 \mu \mathrm{m}$ syringe filters to remove big aggregates and sterilize the solution. The resultant IJA solution was then sub-packed in penicillin bottles for lyophilization. Before use, the IJA powder was resuspended in water or $5 \%$ dextrose solution. To quantify the concentration of IJA, the IJA solution was mixed with equal volume of ethanol and absorption spectra was measured. The concentration of IJA was calculated using a standard concentration-OD curve of ICG.

\section{Photothermal heating experiments in vitro}

$2 \mathrm{~mL}$ IJA or ICG aqueous solution placed in quartz cuvettes, irradiated by continuous-wave diode NIR laser with a wavelength of $808 \pm 5 \mathrm{~nm}$ at the power of $1 \mathrm{~W} / \mathrm{cm}^{2}$ for different time, after which the absorption spectra were acquired. To study the temperature elevation, a fiber optic temperature measuring probe was placed perpendicular to the light path to monitor the temperature in real time. The solution was irradiated with laser for $300 \mathrm{~s}(\mathrm{ON})$ and then cooled for 600s (OFF). The ON/OFF cycle was repeated for four times.

\section{Phantom test}

The Phantom test was conducted using a previously described method [43].

\section{Cell experiments}

Human umbilical vein endothelial cells (HUVECs) and 4T1 murine breast cancer cells were cultured in RPMI-1640 cell culture medium supplemented with $10 \%$ fetal bovine serum (FBS) and $1 \%$ penicillin / streptomycin under the standard cell culture conditions. Cell Counting Kit 8 (CCK8, Dojindo Molecular Technologies, Inc.) assay was carried out following the manufacturer's protocol to determine relative cell viabilities after different treatments.

To study the cytotoxicity, IJA and ICG were firstly dissolved in water at a concentration of 2 $\mathrm{mg} / \mathrm{mL}$ and then diluted in cell culture medium to different concentrations, which were then used to treat HUVECs and 4T1 cancer cells pre-seeded in 96-well plate. After incubated for $24 \mathrm{~h}$, cellular viabilities were determined by CCK8 assay.

To evaluate the disassociation of IJA after cell internalization, 4T1 cells pre-seeded in 96-well plate were firstly incubated with IJA dispersed in cell culture medium at the concentration of $0.1 \mathrm{mg} / \mathrm{mL}$ for 4 hours and washed with PBS for three times. The cells were then incubated in $100 \mu \mathrm{L}$ cell culture medium for $0 \mathrm{~h}, 2 \mathrm{~h}, 4 \mathrm{~h}, 8 \mathrm{~h}, 12 \mathrm{~h}, 16 \mathrm{~h}$ and $20 \mathrm{~h}$, after which the fluorescence images were acquired on an animal fluorescence imaging system. For the control group, 4T1 cells were rinsed with IJA solution, washed with PBS for three times and then incubated in cell culture medium for fluorescence imaging immediately. After fluorescence image acquisition, $100 \mu \mathrm{L}$ DMSO was added for each well to dissolve IJA and fluorescence image were acquired again.

To visualize the photothermal killing effect of IJA, 4T1 cells pre-seeded in 24-well plate were incubated with IJA dispersed in cell culture medium or cell culture medium only. For laser irradiation groups, cells were irradiated with laser at the power of $1 \mathrm{~W} / \mathrm{cm}^{2}$. After incubated for $30 \mathrm{~min}$, cells were stained with Calcein AM and propidium iodide (PI) and then imaged with a fluorescence microscope.

To test the photothermal cytotoxicity, 4T1 cells pre-seeded in 96-well plate were incubated with IJA at different concentrations and then irradiated with laser at the power of $1 \mathrm{~W} / \mathrm{cm}^{2}$ for $5 \mathrm{~min}$. After incubated for another $24 \mathrm{~h}$, cellular viabilities were determined by CCK 8 assay.

\section{Animal experiments}

Female Balb/c mice were purchased from Beijing Vital River Laboratories and all animal experiments were conducted under protocols approved by Peking University Laboratory Animal Center. Xenograft mice models were established by injecting subcutaneously $5-6 \times 10^{6} 4 \mathrm{~T} 1$ cells into the right flank of mouse.

\section{Photoacoustic imaging (PAI) in vivo}

For in vivo PAI tests, 4T1 tumor-bearing mice were intravenously injected with ICG or IJA dispersed in $5 \%$ dextrose solution $(0.2 \mathrm{mg} / \mathrm{mL}, 200 \mu \mathrm{L} /$ mouse $)$. A volume ROI consisting of transverse slices with a step size of $0.3 \mathrm{~mm}$ spanning from liver to tumor region was selected by manual inspection of live MSOT images. Multispectral imaging was performed before injection and $2 \mathrm{~h}, 4 \mathrm{~h}, 8 \mathrm{~h}$ and $24 \mathrm{~h}$ after injection. Images were reconstructed using a model-based approach [12].

\section{Fluorescence imaging}

For fluorescence imaging, 4T1 tumor-bearing mice were intravenously injected with ICG or IJA dispersed in $5 \%$ dextrose solution $(1 \mathrm{mg} / \mathrm{mL}, 200$ $\mu \mathrm{L} /$ mouse). Images were acquired before injection and $2 \mathrm{~h}, 4 \mathrm{~h}, 8 \mathrm{~h}$ and $24 \mathrm{~h}$ post injection. After $24 \mathrm{~h}$, 
mice were euthanized and then tumors were dissected for imaging.

\section{Photothermal therapy (PTT) in vivo}

4T1 tumor-bearing mice were divided into six groups: control groups, laser only, ICG only, IJA only, ICG +laser and IJA+ laser (6 mice each group). In control group and laser only group, mice were injected with $200 \mu \mathrm{L}$ 5\% dextrose solution. Other groups were i.v. injected with IJA or ICG solution dispersed in dispersed in 5\% dextrose solution (1 $\mathrm{mg} / \mathrm{mL}, 200 \mu \mathrm{L} /$ mouse). Laser irradiation groups were irradiated with the $808 \pm 5 \mathrm{~nm}$ laser at a power density of $1 \mathrm{~W} / \mathrm{cm}^{2}$ for $10 \mathrm{~min}$ at $\sim 4$ h post injection. Temperature elevation of tumor area was monitored in a real-time way with an infrared thermal camera. The tumor sizes were measured by a caliper every other day and calculated as the tumor volume = (tumor length) $\times(\text { tumor width })^{2} / 2$. Mice with tumor sizes exceeding $1000 \mathrm{~mm}^{3}$ were euthanatized according to the standard animal protocol. Relative tumor volumes were calculated as $\mathrm{V} / \mathrm{V}_{0}\left(\mathrm{~V}_{0}\right.$ was the tumor volume when the treatment was initiated).

To evaluate the toxicity of IJA and ICG in vivo, 20 days after ICG and IJA administration, vital organs including heart, liver, spleen, lung and kidney of mice were collected and stained using standard hematoxylin \& eosin (H\&E) staining protocol.

\section{Results and Discussions}

\section{Preparation and characterization of IJA}

In aqueous solution, ICG mainly existed in monomers and H-type dimers characterized two absorption peaks at $780 \mathrm{~nm}$ and $\sim 715 \mathrm{~nm}$, respectively (Figure 1A). Under a water bath heating at $65^{\circ} \mathrm{C}$, these two absorption peaks gradually decreased and a new absorption peak at around $895 \mathrm{~nm}$ increased, indicating the transformation of ICG monomers and dimers into J-aggregates, in which the ICG was packed in a head-to-tail manner through the п-п stacking and electrostatic interaction. After heated for around $20 \mathrm{~h}$, the absorption spectra stopped changing, indicating the accomplishment of IJA production. After heating, the IJA solution was dialyzed against water to remove any free ICG. The absorbance was not significantly changed (Figure S1A), indicating that most of the ICG molecules were transformed into IJA. The big J-aggregates were removed by filtration through $0.22 \mu \mathrm{m}$ membrane (Figure S1B). The IJA can be lyophilized and then well-redispersed without any significant changes in absorption spectra (Figure S1C), making IJA able to be stably stored in powder form at room temperature for a very long time. Compared to free ICG, the fluorescence of IJA was fully bleached (Figure S2). Transmission electron microscopy (TEM) images show that the sizes of most IJA are sub-100 nm (Figure 1B). The dynamic light scattering measurements show a narrow size distribution with an average diameter of $\sim 91.7 \mathrm{~nm}$, in accordance with the TEM results (Figure S3). The absorbance of IJA at $895 \mathrm{~nm}$ increases linearly with the concentration, indicating that IJA is not easy to dissociated in water (Figure S4). The IJA could be well dispersed in various media including saline, phosphate buffer saline (PBS), fetal bovine serum (FBS) and RMPI-1640 cell culture medium containing $10 \%$ FBS (Figure 1C). After incubated at $37^{\circ} \mathrm{C}$ for $24 \mathrm{~h}$, no significant changes in absorption spectra were observed, indicating the stability of IJA under physiology conditions. In addition, IJA is formed through the non-covalent interactions among ICG molecules, and thus can be easily disassociated into ICG monomers or dimers again under certain circumstance, such as organic solvents, amphiphilic detergent solution (e.g. Polyoxyethylene-40 stearate (PEG40S), Tween 80, Triton X-100, Pluronic F127(PF127)) (Figure 1D). Thus the facile preparation, nano-size, good stability and ability to be disassociated into ICG indicated the potential of IJA for further biomedical applications.

The strong NIR absorption indicated IJA might be a very good PCA. Equal amount of ICG and IJA have equivalent absorption at $\sim 805 \mathrm{~nm}$. When irradiated with $808 \pm 5 \mathrm{~nm}$ laser at the power of 1 $\mathrm{W} / \mathrm{cm}^{2}$, ICG was quickly bleached by laser (Figure S5). On the contrary, the absorbance of IJA around $\sim 808 \mathrm{~nm}$ decreased in a much slower rate. The photobleaching of ICG have been previously demonstrated as a result of a process involving photo-sensitization to form ${ }^{1} \mathrm{O}_{2}$, dioxetane formation, and then dioxetane cleavage resulting into carbonyl products [44]. By forming nanoparticles, the ICG nanoparticles have a much reduced photobleaching rate compared to the free form [27, 45], since the ICG molecule packed in the core have a much lower possibility to access ${ }^{1} \mathrm{O}_{2}$ generated by photosensitization. However, the photobleaching cannot be totally avoided for IJA, because the IJA is formed totally by ICG and the ICG in the outlayer still have access to ${ }^{1} \mathrm{O}_{2}$. The slower bleaching rate resulted in a much better photothermal effect (Figure 1E). The photothermal conversion ability of IJA does not compromise significantly after multiple cycles of laser-induced photothermal heating. In comparison, the photothermal temperature elevation by ICG diminished sharply after each round of photothermal heating. The enhanced photothermal stability makes IJA a promising PCA. 
A

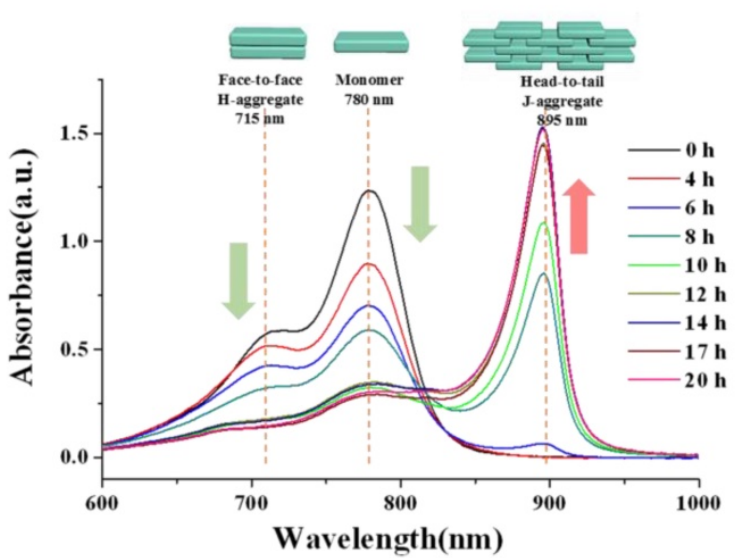

C

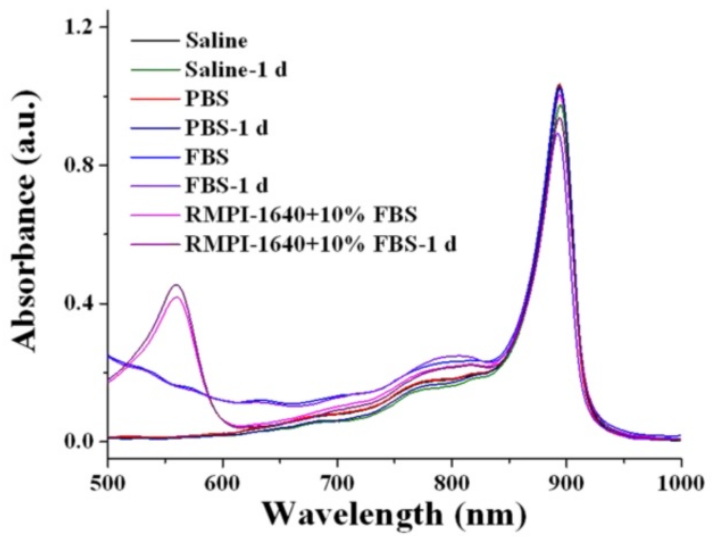

$\mathbf{E}$

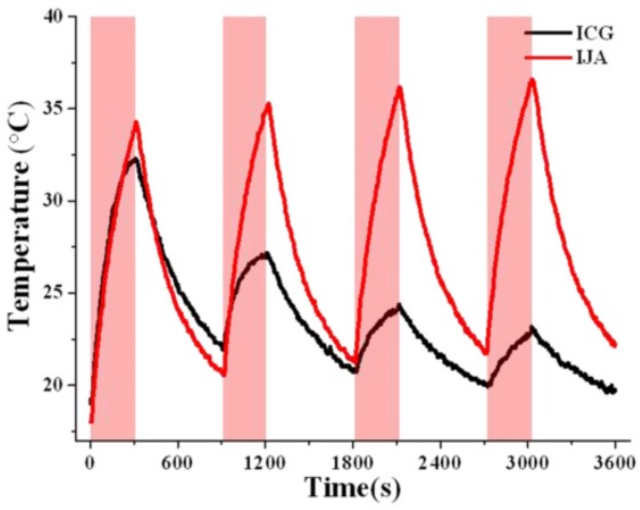

B

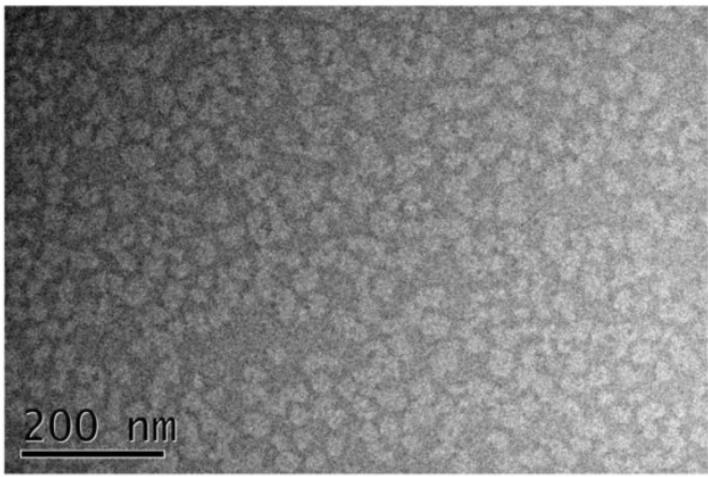

D
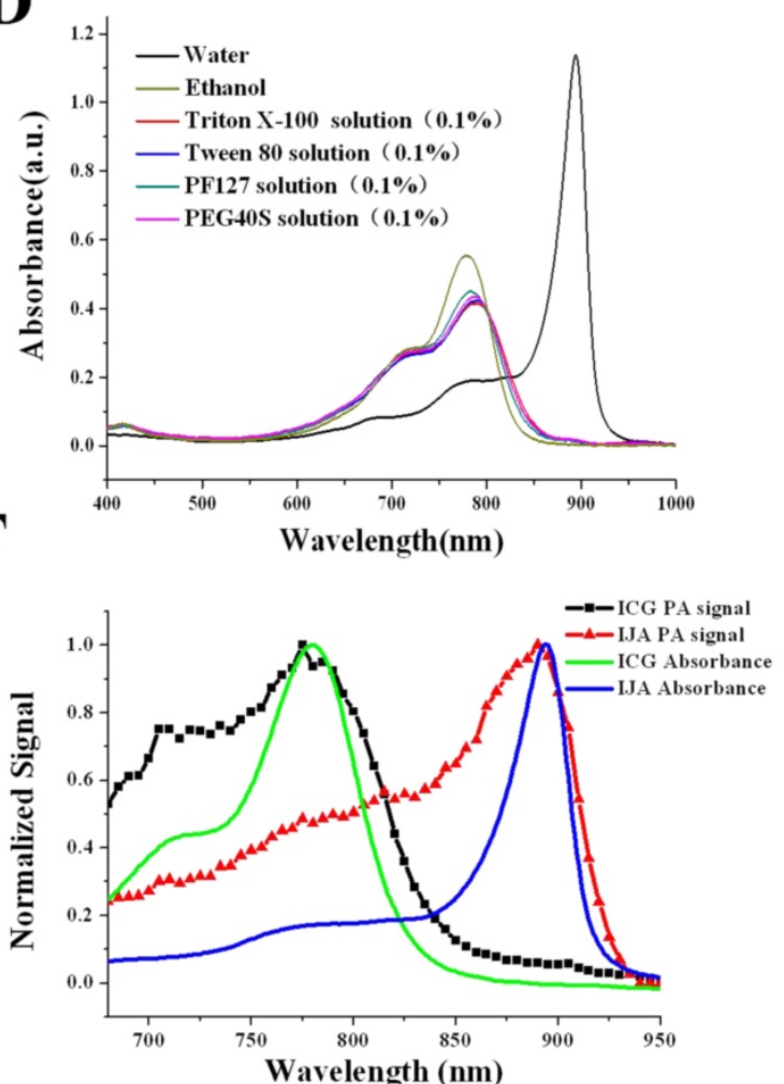

Figure 1. Formation and characterization of IJA in vitro. (A) Absorption spectra indicated the process of IJA formation under heating. (B) TEM image of IJA. (C) Absorption spectra of IJA dispersed in saline, PBS, FBS and RMPI-1640 cell culture medium containing $10 \%$ FBS obtained immediately post-dissolved and after stored for 1 day at $37^{\circ} \mathrm{C}$. (D) Absorption spectra of IJA which was dispersed in water, ethanol, Triton X-100 (1\%, w/w), Tween 80 (1\%, w/w), PF127 (1\%, w/w) and PEG40S $(1 \%, w / w)$, respectively, and then shaken for $2 \mathrm{~h}$ at $37^{\circ} \mathrm{C}$. (E) Temperature changes of IJA and ICG aqueous solution at the concentration of $10 \mu \mathrm{gg} / \mathrm{mL}$ over four laser ON/OFF cycles. The sample was irradiated with a laser at the power of $1 \mathrm{~W} / \mathrm{cm}^{2}$ for $300 \mathrm{~s}$ (ON) followed by natural cooling for $600 \mathrm{~s}$ (OFF). (F) Normalized PA signal of IJA and ICG changes as a function of wavelength obtained with a phantom test. The normalized absorption spectra were used for comparison.

The strong absorption in NIR region also makes IJA a good contrast agent for PAI. Using a tissue-mimicking phantom, the photoacoustic (PA) property of IJA was evaluated using the free ICG solution for comparison. Figure 1F shows the normalized absorption spectra and PA intensity for ICG and IJA. As expected, the PA signal shows a strong correlation with the optical absorption. The slight deviations can be attributed to variations of light energy deposition [46]. Compared with ICG or other ICG-encapsulated nanoparticles [23, 24, 27, 43], one of the biggest advantage of IJA for PAI is that the main PA signal peak red-shifted $\sim 115 \mathrm{~nm}$ from 780 $\mathrm{nm}$ to $895 \mathrm{~nm}$. As demonstrated in many literatures [47], the longer wavelength absorption endows better resolution at deeper tissues. Thus the red-shifted PA signal peak makes IJA an excellent PAI contrast agent, which can be used for monitoring the PTT process of IJA. 

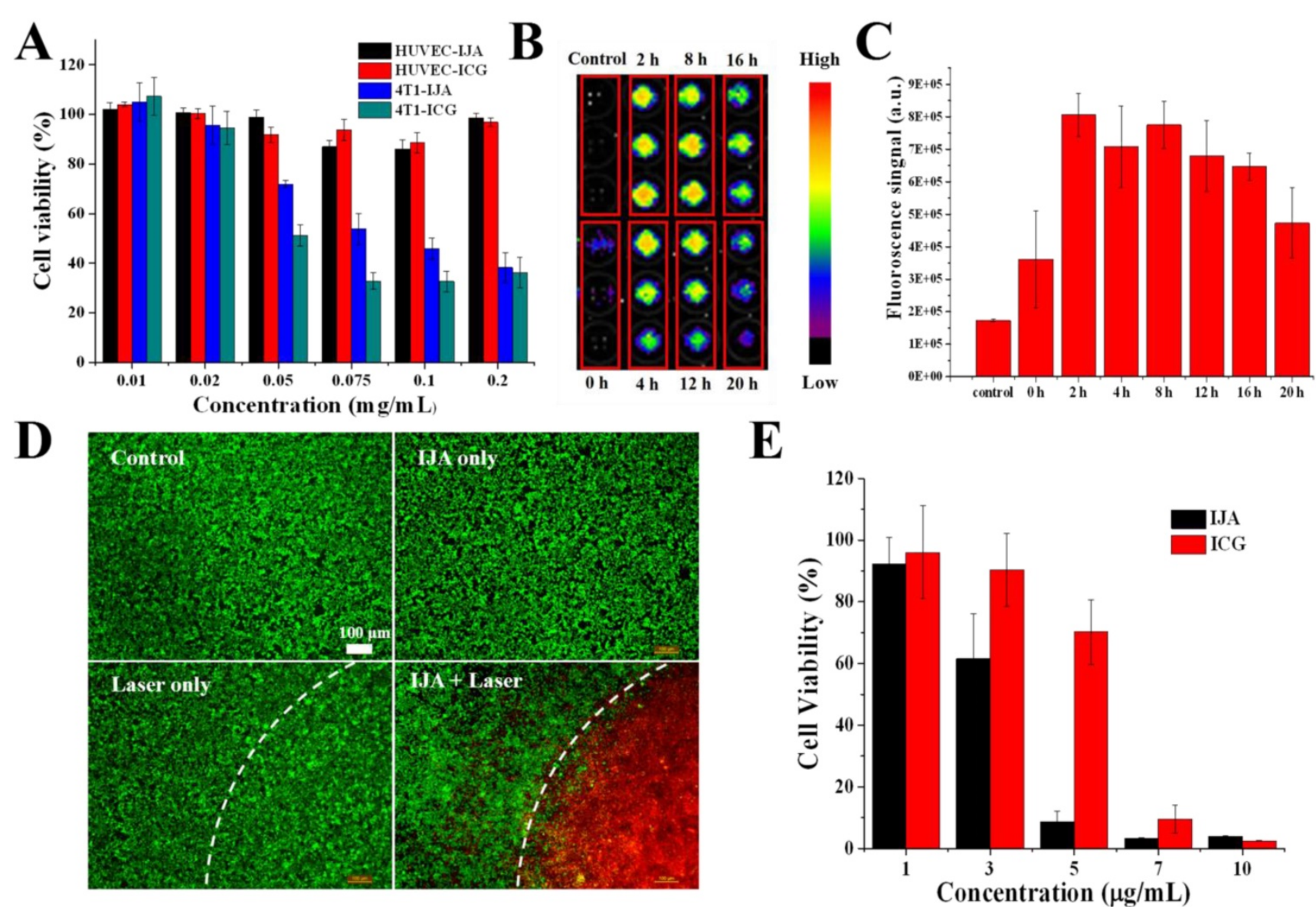

Figure 2. Cell experiments illustrate the non-laser-irradiated and laser-irradiated cytotoxicity in vitro. (A) Cytotoxicity of ICG and IJA to HUVEC and 4TI cancer cell as a function of concentration. (B) $4 \mathrm{~T} 1$ cancer cells were incubated with IJA solution for $4 \mathrm{~h}$ and then incubated in cell culture medium for different time, after which the fluorescence image was acquired. For the control group, cells were just rinsed with IJA solution and then washed off immediately before fluorescence image acquisition. (C) The quantification results based on the fluorescence image. (D) Fluorescence microscopy images of cells under different treatments: no IJA, no laser irradiation(Control); IJA only; Laser irradiation only; IJA treatments and laser irradiation. The cells were stained with Calcein AM (green) and propidium iodide (red) before imaging for visualization of live and dead cells, respectively. (E) Photothermal cytotoxicity of IJA and ICG as a function of concentration.

\section{Cytotoxicity of IJA without / with laser irradiation}

A good biosafety is the prerequisite for further biomedical application in vivo. Using a Cell Counting Kit 8 (CCK8) assay, the cytotoxicity of IJA was evaluated in human umbilical vein endothelial cells (HUVECs) and 4T1 murine breast cancer cells. As shown in Figure 2A, at a concentration as high as 0.02 $\mathrm{mg} / \mathrm{mL}$, no significant cytotoxicity was observed in both cells indicating the biocompatibility of IJA. At even higher concentrations, IJA showed some cytotoxicity to $4 \mathrm{~T} 1$ cancer cell while still negligible cytotoxicity to HUVEC, similar to ICG. The high safety to vascular endothelial cells at very high concentration makes IJA a safe intravenous-injectable agent, since the vascular endothelial cell is one of the primary cell types to contact IJA at such high concentration for systematic administration. Furthermore, we encouragingly found that after internalized by tumor cells IJA can restore the fluorescence again in a few hours, indicating that IJA can be disassociated into ICG monomers in cells (Figure 2B\&C, S6A\&B). From the in vitro experiments, we found that IJA is stable under different physiological conditions but can be dissociated into monomer under hydrophobic or amphiphilic environments. Therefore, the internalization-responsive fluorescence might be a result of existence of amphiphilic substances, such as amphiphilic membrane proteins and lipids. This phenomenon also explained the similar cytotoxicity of IJA and ICG. Since ICG is a FDA-approved drug that has been safely used for many years, it is reasonable to hypothesize that IJA may also be a very safe drug. In addition, since IJA can be disassociated in cells, IJA may also be used as an internalization- responsive fluorescence probe.

The PTT effect of IJA to tumor cells was firstly evaluated at cellular level. As expected, after treated with IJA, most of the tumor cells in the laser-irradiated region were localized killed (Figure 2D). The photothermal cytotoxicity of IJA is concentration-dependent (Figure 2E). At a 
concentration of $5 \mu \mathrm{g} / \mathrm{mL}$, IJA was able to kill over $90 \%$ of cells, while free ICG only induced $\sim 30 \%$ of cells to death. The significant higher PTT effect can be attributed to the reduced photobleaching of IJA. In addition, since nano-sized IJA is expected to have a better tumor accumulation than small molecular ICG, the difference in PTT effect between IJA and ICG may be further enlarged when applied in vivo.

\section{Dual modal imaging in vivo}

To investigate the in vivo biodistribution of IJA, the in vivo PAI was conducted on the 4T1 tumor-bearing mice utilizing a real-time multispectral optoacoustic tomographic (MSOT) imaging system. After injected intravenously at the same dose (2 $\mathrm{mg} / \mathrm{kg}$ ), IJA obviously accumulated in the tumor site, while no significant PA signal in tumor was observed for ICG (Figure 3A). The PA signal of IJA in tumors reaches to maximum at $\sim 4 \mathrm{~h}$ and then gradually decreased, probably due to the dissociation and excretion of IJA (Figure S7A). Similar to ICG, IJA shows significant distribution in liver, spleen and intestines (Figure S8A\&B). In addition, we also found quite strong PA signals of ICG $\sim 8 \mathrm{~h}$ post IJA injection were observed in liver and spleen, indicating that IJA was gradually transformed into ICG in vivo. The excellent tumor accumulation and ability to be disassociated into ICG in vivo makes IJA possible for further image-guided tumor ablation.

The in vivo biodistribution of IJA was also investigated on a fluorescence imaging system utilizing the recovered fluorescence after cellular internalization. As shown in Figure 3B\&S7B, we indeed found that the tumor site was light up by the recovered fluorescence of IJA post injection, demonstrated the accumulation of IJA by the enhanced permeability and retention (EPR) effect. Although PAI results indicated IJA accumulated significantly higher than ICG, the fluorescence of IJA in tumor site is similar to ICG (Figure S9. The possible reason is that most of IJA in tumor is not internalized by tumor cells but retained in the extracellular matrix. The surface negative charge of IJA makes it not easy internalized by cells. For future applications, the combination of fluorescence imaging results and PAI results may be used to evaluate the cellular internalization.

\section{PTT in vivo}

The excellent photothermal conversion ability and tumor accumulation made it possible to use IJA for PTT in vivo. $4 \mathrm{~T} 1$ tumor-bearing mice were divided into six groups: control group, laser-only group, ICG-only group, ICG + laser group, IJA-only group and IJA + laser group (6 mice each group). For control group and laser-only group, $200 \mu \mathrm{L} 5 \%$ dextrose aqueous solution $(\mathrm{w} / \mathrm{w})$ was injected intravenously. Mice in the other groups were injected with IJA or ICG dispersed in 5\% dextrose aqueous solution (1 $\mathrm{mg} / \mathrm{mL}, 200 \mu \mathrm{L}) .4 \mathrm{~h}$ post injection, the mice in laser-irradiation groups were irradiated with an $808 \pm$ $5 \mathrm{~nm}$ laser for 10 minutes. As shown in Figure $\mathbf{4 A \& B}$, Temperature in tumors of IJA + laser group quickly increased to over $55^{\circ} \mathrm{C}$, while the laser only group and ICG + laser group showed a temperature below $45^{\circ} \mathrm{C}$. The higher temperature elevation in IJA + laser group induced the ablation of tumors and prolonged survival time (Figure 4C\&D). In the IJA + laser group, the tumors stopped growing after the treatment and no recurrence was observed during the 20 days. However, for the control groups, the tumor growth is not significantly affected by these treatments. Tumors in the control groups continue growing crazyly and all the mice were dead in two weeks. The excellent PTT performance is the result of the enhanced photothermal conversion ability and tumor accumulation by EPR effect.

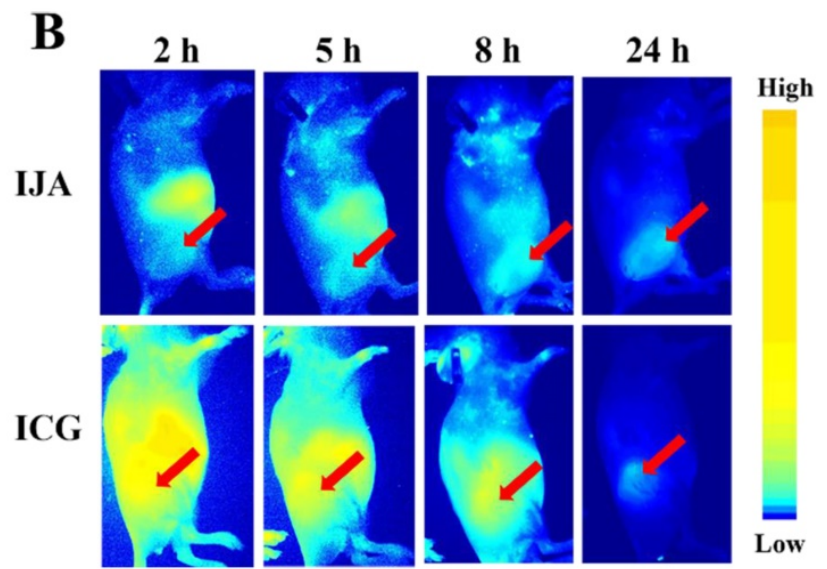

Figure 3. (A) Photoacoustic images of IJA or ICG in 4TI tumor-bearing mice acquired at different time post injection. The tumor regions were indicated in red circles. (B) Fluorescence images acquired at different time following IJA of ICG injection. The red arrows indicated the sites of tumors. 


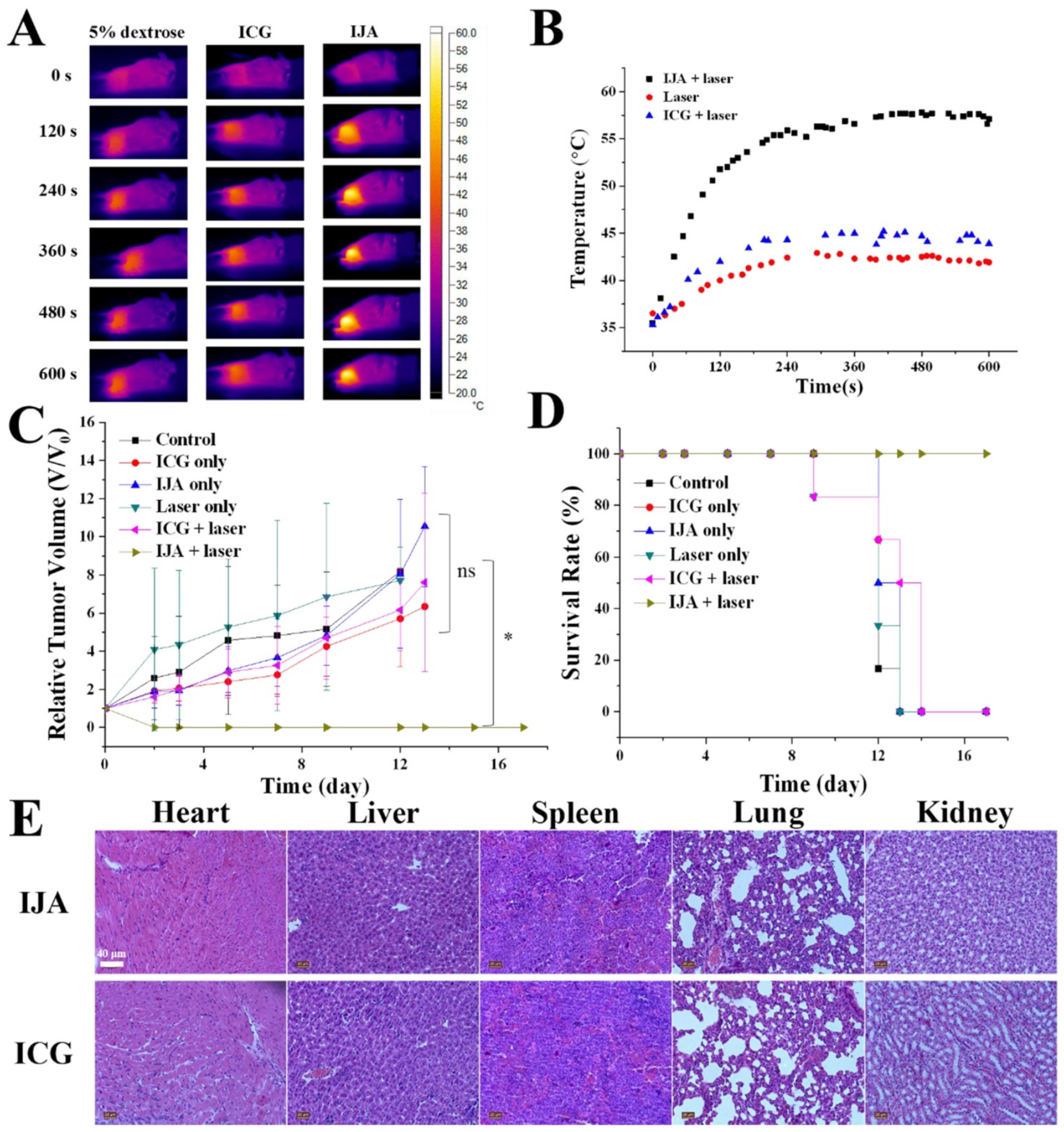

Figure 4. PTT results in 4TI tumor-bearing mice. (A) Thermal images indicated temperature changes of the tumor site under laser irradiation at the power of $1 \mathrm{~W} / \mathrm{cm}^{2}$ for $10 \mathrm{~min}$. (B) Temperature at tumor site changes as a function of time. (C) Time-dependent tumor relative volume curves in different groups (6 mice per group). Correlations analysis between the control groups didn't show statistically significant difference. The difference between control and the experimental group marked with * were statistically significant $(P<0.05)$. (D) The survival curves of mice in different groups. $(E)$ H\&E staining images of major organs collected 20 days post ICG or IJA injection.

Throughout the process for all the groups, no significant changes in body weight were observed (Figure S10). Histological examination of major organs (heart, liver, spleen, lung and kidney) collected 20 days post ICG or IJA administration didn't show any abnormality (Figure 4E). Although further systematic toxicology evaluations are still needed, the preliminary results indicated the biosafety of IJA as an injectable agent.

\section{Conclusion}

In summary, nano-sized IJA were successfully prepared with a very facile method. The IJA is formed by non-covalent packing of ICG molecules and remains in J-aggregated state in various solutions. 
After cellular internalization, IJA can be disassociated into free ICG and exhibit excellent biosafety comparable to ICG. Compared with ICG, IJA showed reduced photobleaching and red-shifted absorption peak, making it a promising agent for PTT and PAI. In addition, the recovery of fluorescence after cellular internalization also makes IJA a promising internalization-responsive fluorescence probe. The facile preparation, high biosafety and excellent theranostic performance makes IJA a promising one-component agent for future biomedical applications. However, admittedly, there are still several issues to deal with. For example, the manufacturing process still needs some optimization to reduce the degradation of ICG during heating and the systematic biosafety still needs much more detailed evaluations.

\section{Acknowledgements}

This work was financially supported by National Key Research and Development Program of China (No. 2016YFA0201400), State Key Program of National Natural Science of China (Grant No. 81230036), National Natural Science Foundation of China (No. 81371580) and the Foundation for Innovative Research Groups of the National Natural Science Foundation of China (No. 81421004).

\section{Supplementary Material}

Supplementary figures.

http://www.ntno.org/v01p0430s1.pdf

\section{Competing Interests}

The authors have declared that no competing interest exists.

\section{References}

1. Skinner MG, Iizuka MN, Kolios MC, Sherar MD. A theoretical comparison of energy sources-microwave, ultrasound and laser-for interstitial thermal therapy. Physics in medicine and biology. 1998; 43: 3535.

2. Stauffer PR. Evolving technology for thermal therapy of cancer. International Journal of Hyperthermia. 2005; 21: 731-44.

3. Ke $\mathrm{H}$, Wang J, Dai $\mathrm{Z}$, Jin $\mathrm{Y}, \mathrm{Qu}$ E, Xing $\mathrm{Z}$, et al. Gold-Nanoshelled Microcapsules: A Theranostic Agent for Ultrasound Contrast Imaging and Photothermal Therapy. Angewandte Chemie. 2011; 123: 3073-7.

4. Huang P, Rong P, Lin J, Li W, Yan X, Zhang MG, et al. Triphase interface synthesis of plasmonic gold bellflowers as near-infrared light mediated acoustic and thermal theranostics. Journal of the American Chemical Society. 2014; 136: 8307-13

5. Huang X, Tang S, Mu X, Dai Y, Chen G, Zhou Z, et al. Freestanding palladium nanosheets with plasmonic and catalytic properties. Nature nanotechnology. 2011; 6: 28-32.

6. Chen M, Tang S, Guo Z, Wang X, Mo S, Huang X, et al. Core-Shell Pd@ Au Nanoplates as Theranostic Agents for In-Vivo Photoacoustic Imaging, CT Imaging, and Photothermal Therapy. Advanced Materials. 2014; 26: 8210-6.

7. Li M, Li L, Zhan C, Kohane DS. Core-Shell Nanostars for Multimodal Therapy and Imaging. Theranostics. 2016; 6: 2306.

8. Jin Y, Wang J, Ke H, Wang S, Dai Z. Graphene oxide modified PLA microcapsules containing gold nanoparticles for ultrasonic/CT bimodal imaging guided photothermal tumor therapy. Biomaterials. 2013; 34: 4794-802.

9. Moon $\mathrm{HK}$, Lee $\mathrm{SH}$, Choi $\mathrm{HC}$. In vivo near-infrared mediated tumor destruction by photothermal effect of carbon nanotubes. ACS nano. 2009; 3: 3707-13
10. Chen D, Dougherty CA, Zhu K, Hong H. Theranostic applications of carbon nanomaterials in cancer: Focus on imaging and cargo delivery. Journal of Controlled Release. 2015; 210: 230-45.

11. Yang K, Zhang S, Zhang G, Sun X, Lee S-T, Liu Z. Graphene in mice: ultrahigh in vivo tumor uptake and efficient photothermal therapy. Nano letters. 2010; 10: 3318-23.

12. Liu R, Jing L, Peng D, Li Y, Tian J, Dai Z. Manganese (II) chelate functionalized copper sulfide nanoparticles for efficient magnetic resonance/photoacoustic dual-modal imaging guided photothermal therapy. Theranostics. 2015; 5: 1144.

13. Cheng L, Liu J, Gu X, Gong H, Shi X, Liu T, et al. PEGylated WS2 Nanosheets as a Multifunctional Theranostic Agent for in vivo Dual-Modal CT/Photoacoustic Imaging Guided Photothermal Therapy. Advanced Materials. 2014; 26: 1886-93.

14. Wang J, Pang X, Tan X, Song Y, Liu L, You Q, et al. A triple-synergistic strategy for combinational photo/radiotherapy and multi-modality imaging based on hyaluronic acid-hybridized polyaniline-coated WS 2 nanodots. Nanoscale. 2017; 9: 5551-64.

15. Zha Z, Yue X, Ren Q, Dai Z. Uniform polypyrrole nanoparticles with high photothermal conversion efficiency for photothermal ablation of cancer cells. Advanced materials. 2013; 25: 777-82.

16. Xu L, Cheng L, Wang C, Peng R, Liu Z. Conjugated polymers for photothermal therapy of cancer. Polymer Chemistry. 2014; 5: 1573-80.

17. Yang K, Li Y, Tan X, Peng R, Liu Z. Behavior and toxicity of graphene and its functionalized derivatives in biological systems. Small. 2013; 9: 1492-503.

18. Khlebtsov N, Dykman L. Biodistribution and toxicity of engineered gold nanoparticles: a review of in vitro and in vivo studies. Chemical Society Reviews. 2011; 40: 1647-71.

19. Hao J, Song G, Liu T, Yi X, Yang K, Cheng L, et al. In Vivo Long-Term Biodistribution, Excretion, and Toxicology of PEGylated Transition-Metal Dichalcogenides MS2 (M= Mo, W, Ti) Nanosheets. Advanced Science. 2016.

20. Muthu MS, Leong DT, Mei L, Feng S-S. Nanotheranostics-application and further development of nanomedicine strategies for advanced theranostics. Theranostics. 2014; 4: 660-77.

21. Wang J, Guo F, Yu M, Liu L, Tan F, Yan R, et al. Rapamycin/DiR loaded lipid-polyaniline nanoparticles for dual-modal imaging guided enhanced photothermal and antiangiogenic combination therapy. Journal of Controlled Release. 2016; 237: 23-34

22. Morton JG, Day ES, Halas NJ, West JL. Nanoshells for photothermal cancer therapy. Cancer nanotechnology: methods and protocols. 2010: 101-17.

23. Yan F, Wu H, Liu H, Deng Z, Liu H, Duan W, et al. Molecular imaging-guided photothermal/photodynamic therapy against tumor by iRGD-modified indocyanine green nanoparticles. Journal of Controlled Release. 2016; 224: 217-28.

24. Ma Y, Tong S, Bao G, Gao C, Dai Z. Indocyanine green loaded SPIO nanoparticles with phospholipid-PEG coating for dual-modal imaging and photothermal therapy. Biomaterials. 2013; 34: 7706-14.

25. Zheng M, Yue C, Ma Y, Gong P, Zhao P, Zheng C, et al Single-step assembly of DOX/ICG loaded lipid-polymer nanoparticles for highly effective chemo-photothermal combination therapy. ACS nano. 2013; 7: 2056-67.

26. Navarro FP, Berger M, Guillermet S, Josserand V, Guyon L, Neumann E, et al. Lipid nanoparticle vectorization of indocyanine green improves fluorescence imaging for tumor diagnosis and lymph node resection. Journal of biomedical nanotechnology. 2012; 8: 730-41.

27. Sheng $\mathrm{Z}, \mathrm{Hu} \mathrm{D}$, Zheng $\mathrm{M}$, Zhao $\mathrm{P}$, Liu $\mathrm{H}$, Gao $\mathrm{D}$, et al Smart human serum albumin-indocyanine green nanoparticles generated by programmed assembly for dual-modal imaging-guided cancer synergistic phototherapy. ACS nano. 2014; 8: 12310-22.

28. Chen $\mathrm{Q}$, Liang $\mathrm{C}$, Wang $X, \mathrm{He} J, \mathrm{Li} Y$, Liu Z An albumin-based theranostic nano-agent for dual-modal imaging guided photothermal therapy to inhibit lymphatic metastasis of cancer post surgery. Biomaterials. 2014; 35: 9355-62.

29. Huang $\mathrm{P}, \mathrm{Gao} Y$, Lin J, Hu H, Liao $\mathrm{H}-\mathrm{S}$, Yan $\mathrm{X}$, et al. Tumor-specific formation of enzyme-instructed supramolecular self-assemblies as cancer theranostics. ACS nano. 2015; 9: 9517-27.

30. Jelley EE. Spectral absorption and fluorescence of dyes in the molecular state. Nature $1936 ; 138: 1009-10$.

31. Eisfeld A, Briggs J. The J-and H-bands of organic dye aggregates. Chemical Physics. 2006; 324: 376-84.

32. Spano FC, Mukamel S. Superradiance in molecular aggregates. The Journal of Chemical Physics. 1989; 91: 683-700.

33. Lidzey D, Tartakovskii A, Emam-Ismail M, Skolnick M, Walker S. Enhanced Raman scattering in a strongly coupled microcavity containing J-aggregates. Synthetic metals. 2002; 127: 151-4.

34. Dai Z, Tian W, Yue X, Zheng Z, Qi J, Tamai N, et al. Efficient fluorescence resonance energy transfer in highly stable liposomal nanohybrid cerasome. Chemical Communications. 2009: 15.2032-2034

35. Lovell JF, Jin CS, Huynh E, Jin H, Kim C, Rubinstein JL, et al. Porphysome nanovesicles generated by porphyrin bilayers for use as multimodal biophotonic contrast agents. Nat Mater. 2011; 10: 324-32.

36. Shakiba M, Ng KK, Huynh E, Chan H, Charron DM, Chen J, et al. Stable J-aggregation enabled dual photoacoustic and fluorescence nanoparticles for intraoperative cancer imaging. Nanoscale. 2016; 8: 12618-25.

37. Song X, Gong H, Liu T, Cheng L, Wang C, Sun X, et al. J-aggregates of organic dye molecules complexed with iron oxide nanoparticles for imaging-guided photothermal therapy under 915-nm light. Small. 2014; 10: 4362-70. 
38. Song $\mathrm{X}$, Zhang R, Liang $\mathrm{C}$, Chen $\mathrm{Q}$, Gong $\mathrm{H}$, Liu Z. Nano-assemblies of J-aggregates based on a NIR dye as a multifunctional drug carrier for combination cancer therapy. Biomaterials. 2015; 57: 84-92.

39. Rotermund F, Weigand R, Penzkofer A. J-aggregation and disaggregation of indocyanine green in water. Chemical physics. 1997; 220: 385-92.

40. Zweck J, Penzkofer A. Microstructure of indocyanine green J-aggregates in aqueous solution. Chemical Physics. 2001; 269: 399-409.

41. Yamauchi F, Takahashi A, Kanazaki K, Ogawa S, Minami M, Sasaguri D, et al. Indocyanine green-containing particle and contrast agent for photoacoustic imaging having the particle. Google Patents; 2013.

42. Wittmann M, Rotermund F, Weigand R, Penzkofer A. Saturable absorption and absorption recovery of indocyanine green J-aggregates in water. Applied Physics B: Lasers and Optics. 1998; 66: 453-9.

43. Beziere N, Lozano N, Nunes A, Salichs I, Queiros D, Kostarelos K, et al. Dynamic imaging of PEGylated indocyanine green (ICG) liposomes within the tumor microenvironment using multi-spectral optoacoustic tomography (MSOT). Biomaterials. 2015; 37: 415-24.

44. Gorka AP, Nani RR, Schnermann MJ. Cyanine polyene reactivity: scope and biomedical applications. Organic \& biomolecular chemistry. 2015; 13: 7584-98.

45. Huang P, Rong P, Jin A, Yan X, Zhang MG, Lin J, et al. Dye-Loaded Ferritin Nanocages for Multimodal Imaging and Photothermal Therapy. Advanced Materials. 2014; 26: 6401-8.

46. Ho CJ, Balasundaram G, Driessen W, McLaren R, Wong CL, Dinish US, et al. Multifunctional photosensitizer-based contrast agents for photoacoustic imaging. Sci Rep. 2014; 4: 5342.

47. Zhou Y, Wang DP, Zhang YM, Chitgupi U, Geng JM, Wang YH, et al. A Phosphorus Phthalocyanine Formulation with Intense Absorbance at $1000 \mathrm{~nm}$ for Deep Optical Imaging. Theranostics. 2016; 6: 688-97. 